Gradient estimates in Morrey spaces of weak solutions to quasilinear parabolic systems of Hörmander's vector fields

\author{
Yan Dong
}




\title{
GRADIENT ESTIMATES IN MORREY SPACES OF WEAK SOLUTIONS TO QUASILINEAR PARABOLIC SYSTEMS OF HÖRMANDER'S VECTOR FIELDS
}

\author{
YAN DONG
}

Received 16 April, 2013

\begin{abstract}
This paper is concerned with higher integrability for gradients of weak solutions to quasilinear parabolic systems of Hörmander's vector fields. We establish $L^{p}$ estimates for gradients of weak solutions by deriving a parabolic Caccioppli inequality and using the reverse Hölder inequality in parabolic cylinders, and then obtain $L^{p}$ estimates for gradients of weak solutions to homogeneous parabolic system. At last higher integrability of gradients in Morrey space with $p \geq 2$ is proved.
\end{abstract}

2010 Mathematics Subject Classification: 35K55; 35B65

Keywords: quasilinear parabolic system, Hörmander's vector fields, higher integrability, Caccioppoli inequality, Morrey space, natural condition

\section{INTRODUCTION}

Let $\mathrm{X}=\left(X_{1}, \cdots, X_{q}\right)$ be a family of real smooth vector fields in a neighborhood $\tilde{\Omega}$ of some bounded domain $\Omega \subset \mathrm{R}^{n}(q \leq n)$ with the form

$$
X_{j}=\sum_{k=1}^{n} b_{j k}(x) \frac{\partial}{\partial x_{k}}, \quad\left(b_{j k}(x) \in C^{\infty}(\Omega), j=1,2, \ldots, q\right)
$$

and satisfying Hörmander's condition, namely, the Lie algebra generated by the family $X_{1}, \ldots, X_{q}$ at any point of $\tilde{\Omega}$ spans $\mathbb{R}^{n}$.

Regularity for weak solutions to parabolic equations and systems in Euclidean spaces (i.e., $q=n, X_{i}=\frac{\partial}{\partial x_{i}}, i=1,2, \cdots, n$ ) had fairly complete results. When coefficients are discontinuous and belong to some VMO spaces, Mcbride in [15] derived the generalized Morrey estimates for gradients of weak solutions to linear parabolic systems. Giaquinta and Struwe in [12] treated partial regularity for weak solutions to quasilinear parabolic systems under the natural condition and got a sharp Hölder

This work was supported by the National Natural Science Foundation of China (Grant Nos. 11271299, 11001221), Natural Science Foundation Research Project of Shaanxi Province (2012JM1014) and NPU Foundation for Foundmental Research(NPU-FFR-JC20110271). 
exponent by establishing a Caccioppoli inequality and the reverse Hölder inequality. For some other studies, we quote $[2,3,11,17]$ and references therein.

Divergence equations or systems constituted by Hörmander's vector fields have received extensive attention. Di Fazio and Fanciullo in [4] studied gradient estimates in Morrey spaces when $p=2$ for weak solutions to linear degenerate elliptic system with coefficients in some VMO spaces. Dong and Niu in [6] generalized the result and derived gradient estimates in Morrey spaces when $p>2$ for weak solutions to linear elliptic systems by establishing energy estimate and using the reverse Hölder inequality on the homogeneous spaces, and got Hölder regularity. Xu and Zuily in [20] attained interior regularity for weak solutions to quasilinear elliptic systems with natural condition. Partial Hölder regularity for weak solutions to quasilinear elliptic systems under quadratic natural growth condition and controllable growth condition was deirved by Gao and Niu in [8]. Related results can also be found in [18, 19].

The purpose of this paper is to investigate quasilinear parabolic systems of Hörmander's vector fields

$$
u_{t}^{i}+X_{\alpha}^{*}\left(a_{i}^{\alpha \beta}(z, u) X_{\beta} u^{i}\right)=g_{i}(z, u, X u)+X_{\alpha}^{*} f_{i}^{\alpha}(z),
$$

where $\alpha, \beta=1,2, \ldots, q, i=1,2, \ldots, N, X_{j}^{*}=-X_{j}+c_{j}\left(c_{j}=-\sum_{k=1}^{n} \frac{\partial b_{j k}}{\partial x_{k}} \in C^{\infty}(\Omega)\right)$ is the transposed vector field of $X_{j}, z=(x, t) \in Q_{T} \subset \mathbb{R}^{n+1}, Q_{T}=\Omega \times(0, T)$, $g_{i}(z, u, X u)$ satisfies natural growth condition. We will establish higher integrability of gradients in the Morrey spaces $L_{X}^{p, \lambda}(p \geqslant 2)$ for weak solutions to (1.1).

Definition 1 (BMO and VMO spaces). For any $f \in L^{1}\left(Q_{T}\right)$, we set

$$
\eta_{R}(f)=\sup _{z_{0} \in Q_{T}, 0 \leqslant \rho \leqslant R}\left(\frac{1}{\left|Q_{T} \cap Q_{\rho}\left(z_{0}\right)\right|} \iint_{Q_{T} \cap Q_{\rho}\left(z_{0}\right)}\left|f(z)-f_{Q_{T} \cap Q_{\rho}\left(z_{0}\right)}(z)\right| d z\right),
$$

where $f_{Q_{T} \cap Q_{\rho}\left(z_{0}\right)}=\frac{1}{\left|Q_{T} \cap Q_{\rho}\left(z_{0}\right)\right|} \iint_{Q_{T} \cap Q_{\rho}\left(z_{0}\right)} f(z) d z$, and the meaning of $Q_{\rho}\left(z_{0}\right)$ sees the next section. If $\sup _{R \rightarrow 0} \eta_{R}(f)<+\infty$, we say $f \in \operatorname{BMO}\left(Q_{T}\right)$ (Bounded Mean Oscillation). Moreover, if $\eta_{R}(f) \rightarrow 0$ as $R \rightarrow 0$, then we say $f \in \operatorname{VMO}\left(Q_{T}\right)$ (Vanishing Mean Oscillation).

Now we introduce the relevant assumptions.

(H1) (ellipticity condition) Let coefficients $a_{i}^{\alpha \beta}(z, u)$ satisfy the ellipticity condition, namely, there exists a constant $\delta>0$ such that for any $\xi \in \mathbb{R}^{(q+1) N}$,

$$
a_{i}^{\alpha \beta}(z, u) \xi_{\alpha}^{i} \xi_{\beta}^{i} \geqslant \delta|\xi|^{2},
$$

where $z=(x, t) \in Q_{T}, Q_{T}=\Omega \times(0, T), \Omega \subset \mathbb{R}^{n}$. 
(H2) $\left(V M O \cap L^{\infty}\right)$ Assume $a_{i}^{\alpha \beta}\left(z, u_{0}\right) \in V M O$ uniformly on $z \in Q_{T}$ for some fixed $u_{0} \in \mathbb{R}^{N}$, that is,

$$
\lim _{R \rightarrow 0} \eta_{R}\left(a_{i}^{\alpha \beta}\left(\cdot, u_{0}\right)\right)=0,
$$

and there exists a constant $L>0$, such that for any $z \in Q_{T}$ and $u \in \mathbb{R}^{N}$, one has $\left|a_{i}^{\alpha \beta}(z, u)\right| \leqslant L$.

(H3) (Continuity) There exist a positive constant $c$ and a continuous concave function $\omega: \mathbb{R}^{+} \rightarrow \mathbb{R}^{+}, \omega(0)=0,0 \leqslant \omega \leqslant 1$, such that for any $z \in Q_{T}, u, v \in \mathbb{R}^{N}$,

$$
\left|a_{i}^{\alpha \beta}(z, u)-a_{i}^{\alpha \beta}(z, v)\right| \leqslant c \omega\left(|u-v|^{2}\right) .
$$

(H4) (Natural growth condition) Let $u \in W_{2}^{1,1}\left(Q_{T}, \mathbb{R}^{N}\right) \cap L^{\infty}\left(Q_{T}, \mathbb{R}^{N}\right)$ (the definition of $W_{2}^{1,1}\left(Q_{T}, \mathbb{R}^{N}\right)$ sees Section 2$), M=\sup _{z \in Q_{T}}|u(z)|$ and $g_{i}(z, u, X u)$ satisfy

$$
\left|g_{i}(z, u, X u)\right| \leqslant \mu(M)\left(|X u|^{2}+g^{i}(z)\right),
$$

where $\mu(M)>0, g^{i}(z) \in L_{X}^{m, \lambda}\left(Q_{T}\right), f_{i}^{\alpha}(z) \in L_{X}^{m, \lambda}\left(Q_{T}\right), i=1,2, \cdots, N, m>$ $p$ (for definition of $p$ see Theorem 3.2), $0<\lambda<Q+2$ ( $Q$ is the homogeneous dimension relative to $\Omega$ ), and $2 \mu(M) M<\delta$.

Definition 2. If $u \in W_{2}^{1,1}\left(Q_{T}, \mathbb{R}^{N}\right) \cap L^{\infty}\left(Q_{T}, \mathbb{R}^{N}\right)$ satisfies

$\iint_{Q_{T}}\left[u_{t}^{i} \varphi^{i}+a_{i}^{\alpha \beta}(z, u) X_{\alpha} \varphi^{i} X_{\beta} u^{i}\right] d z=\iint_{Q_{T}}\left[g_{i}(z, u, X u) \varphi^{i}+f_{i}^{\alpha}(z) X_{\alpha} \varphi^{i}\right] d z$, for any $\varphi \in C_{0}^{\infty}\left(Q_{T}, \mathbb{R}^{N}\right)$, then $u$ is said a weak solution to (1.1).

Now the main result of the paper is stated as follows.

Theorem 1. Suppose that assumptions (H1)-(H4) hold. If $u \in W_{2}^{1,1}\left(Q_{T}, \mathbb{R}^{N}\right) \cap$ $L^{\infty}\left(Q_{T}, \mathbb{R}^{N}\right)$ is a weak solution of $(1.1)$ in $Q_{T}$, then there exists a constant $\varepsilon_{0}>0$ such that for any $p \in\left[2,2+\frac{2 Q}{Q+2} \varepsilon_{0}\right), Q^{\prime} \subset \subset Q^{\prime \prime} \subset \subset Q_{T}$, we have $X u \in L_{X, l o c}^{p, \lambda}\left(Q_{T}\right)$ and

$$
\|X u\|_{L_{X}^{p, \lambda}\left(Q^{\prime}\right)} \leqslant c\left(\|X u\|_{L^{p}\left(Q^{\prime \prime}\right)}+\sum_{i}\left\|g^{i}\right\|_{L_{X}^{p, \lambda}\left(Q_{T}\right)}+\|f\|_{L_{X}^{p, \lambda}\left(Q_{T}\right)}\right) .
$$

Remark 1. These conclusions are new even in quasilinear parabolic systems under Euclidean spaces. The proofs depend on some a priori estimates, e.g., parabolic Caccioppoli inequalities. We note that in the study of regularity to parabolic equations in Euclidean spaces, a normal tool is a parabolic Poincaré inequality. But for (1.1), a corresponding parabolic Poincare inequality is still not proved, we introduce the average $\bar{u}(t)$ of $u(x, t)$ as done in [5] and [12], and apply (2.2) below to deduce a 
parabolic Caccioppoli inequality. In order to get higher integrability, we employ a reverse Hölder inequality in parabolic cylinders.

The plan of the paper is organized as follows. In Section 2, we describe the notions of Carnot-Carathéodory distance, ball, doubling property, parabolic cylinder, and some function spaces related to vector fields, such as Morrey space, Sobolev space, and then recall the known iterative lemma, Sobolev-Poincaré inequality in $\Omega$, reverse Hölder inequality relate to parabolic cylinder. In Section 3, we establish a parabolic Caccioppoli inequality with the average $\bar{u}(t)$ of $u(x, t)$ and get the $L^{p}$ estimates of gradients for weak solutions (Theorem 3) by using the reverse Hölder inequality. In Section 4, higher integrability of gradients for weak solutions to homogeneous parabolic system are derived. In Section 5, the proof of Theorem 1 is given with the help of previous results.

\section{PReliminaries}

Let us denote the commutator of vector fields $X_{1}, \cdots, X_{q}$ by

$$
X_{\beta}=\left[X_{\beta_{d}},\left[X_{\beta_{d-1}}, \cdots\left[X_{\beta_{2}}, X_{\beta_{1}}\right] \cdots\right]\right],
$$

where $\beta=\left(\beta_{1}, \cdots, \beta_{d}\right), 1 \leqslant \beta_{i} \leqslant q, i=1, \cdots, d,|\beta|=d$, and recall that $d$ is the length of $X_{\beta}$.

Definition 3. If $\left\{X_{\beta}\left(x_{0}\right)\right\}_{|\beta| \leqslant s}$ spans $\mathbb{R}^{n}$ at every $x_{0} \in \Omega \subset \mathbb{R}^{n}$, then we say that the system $X=\left(X_{1}, \cdots, X_{q}\right)$ satisfies Hörmander's condition of step $s$.

Following [20], we can assume that Hörmander's vector fields $X_{1}, \cdots, X_{q}$ are free up to the order $s$.

Definition 4 (Carnot-Carathéodory distance). Let $\Omega$ be a bounded set in $\mathbb{R}^{n}$. An absolutely continuous curve $\gamma:[0, T] \rightarrow \Omega$ is called a sub-unit curve with respect to the system $X=\left(X_{1}, \cdots, X_{q}\right)$, if $\gamma^{\prime}(t)$ exists for a.e. $t \in[0, T]$ and satisfies

$$
<\gamma^{\prime}(t), \xi>^{2} \leqslant \sum_{j=1}^{q}<X_{j}(\gamma(t)), \xi>^{2}, \quad \text { for any } \xi \in \mathbb{R}^{n}
$$

We denote the length of this curve by $l_{S}(\gamma)=T$. Given any $x, y \in \Omega$, let $\Phi(x, y)$ be the collection of all sub-unit curves connecting $x$ and $y$, and define the CarnotCarathéodory distance induced by $X$ as follows

$$
d_{\mathrm{X}}(x, y)=\inf \left\{l_{S}(\gamma): \gamma \in \Phi(x, y)\right\} .
$$

With this distance, we denote a metric ball of radius $R$ centered at $x$ by

$$
B_{R}(x)=B(x, R)=\{y \in \Omega: d(x, y)<R\} .
$$

If one does not need to consider the center of the ball, then we also write $B_{R}$ instead of $B(x, R)$. 
It is well known that the doubling property (see [16]) for metric balls holds true, i.e., there exist positive constants $c_{D}$ and $R_{D}$, such that for any $x_{0} \in \Omega, 0<2 R<R_{D}$, $B\left(x_{0}, 2 R\right) \subset \Omega$,

$$
\left|B\left(x_{0}, 2 R\right)\right| \leqslant c_{D}\left|B\left(x_{0}, R\right)\right| .
$$

Furthermore, it follows that for any $R \leqslant R_{D}$ and $\tau \in(0,1)$,

$$
\left|B_{\tau R}\right| \geqslant c_{D}^{-1} \tau^{Q}\left|B_{R}\right| \text {. }
$$

The number $Q=\log _{2} c_{D}$ is called a locally homogeneous dimension relative to $\Omega$. Clearly, $Q \geqslant n$.

As in [20] we assume that for any small positive $R$ there exist two positive constants $c_{1}$ and $c_{2}$, such that

$$
c_{1} R^{Q} \leqslant\left|B_{R}\right| \leqslant c_{2} R^{Q} .
$$

Throughout this paper, we denote $z_{0}=\left(x_{0}, t_{0}\right) \in Q_{T} \subset \mathbb{R}^{n+1}$. A parabolic cylinder with vertex at $z_{0}$ is defined by

$$
Q_{R}\left(z_{0}\right)=B_{R}\left(x_{0}\right) \times\left(t_{0}-\frac{R^{2}}{2}, t_{0}+\frac{R^{2}}{2}\right]
$$

In what follows, let us denote $I_{R}\left(t_{0}\right)=\left(t_{0}-\frac{R^{2}}{2}, t_{0}+\frac{R^{2}}{2}\right]$, and the parabolic boundary of $Q_{R}$ by

$$
\partial_{p} Q_{R}\left(z_{0}\right)=\left(\partial B_{R}\left(x_{0}\right) \times\left(t_{0}-\frac{R^{2}}{2}, t_{0}+\frac{R^{2}}{2}\right]\right) \cup B_{R}\left(x_{0}\right) \times\left\{t_{0}-\frac{R^{2}}{2}\right\} .
$$

We denote by $|B(x, R)|$ the Lebesgue measure of $B(x, R)$ in the $n$-dimensional space, and the Lebesgue measure of $Q_{R}\left(z_{0}\right)$ in the $(n+1)$-dimensional space by $\left|Q_{R}\left(z_{0}\right)\right|$. To simplify the notations, in the sequel, $Q_{R}\left(z_{0}\right), B_{R}\left(x_{0}\right), I_{R}\left(t_{0}\right)$, $\sqrt{\sum_{i=1}^{q}\left|X_{i} u\right|^{2}}$ and $(x, t)$ are written as $Q_{R}, B_{R}, I_{R},|X u|$ and $z$, respectively.

Definition 5 (Morrey space $L_{X}^{p, \lambda}\left(Q_{T}\right)$ ). For $1 \leqslant p<+\infty, \lambda>0$, we say that $f \in L^{p}\left(Q_{T}\right)$ belongs to the Morrey space $L_{X}^{p, \lambda}\left(Q_{T}\right)$ if

$$
\|f\|_{L_{X}^{p, \lambda}}=\sup _{z_{0} \in Q_{T}, 0 \leqslant \rho \leqslant d_{0}}\left(\frac{\rho^{\lambda}}{\left|Q_{T} \cap Q_{\rho}\left(z_{0}\right)\right|} \iint_{Q_{T} \cap Q_{\rho}\left(z_{0}\right)}|f|^{p} d z\right)^{\frac{1}{p}}<\infty,
$$

where $d_{0}$ is the diameter of $Q_{T}$.

It is easy to prove as in [15] that the spaces $L_{X}^{p, \lambda}\left(Q_{T}\right)$ are Banach spaces.

Definition 6 (parabolic Sobolev space $W_{p}^{m, k}\left(Q_{T}\right)$ ). Let $m$ and $k$ be non-negative integers, $1 \leqslant p<+\infty$. The set

$$
W_{p}^{m, k}\left(Q_{T}\right)=\left\{u ; X_{\alpha} u, \partial_{t}^{r} u \in L^{p}\left(Q_{T}\right), 0 \leqslant|\alpha| \leqslant m, 0 \leqslant r \leqslant k\right\}
$$


is called a parabolic Sobolev space related to Hörmander's vector fields, with the norm

$$
\|u\|_{W_{p}^{m, k}\left(Q_{T}\right)}=\sum_{|\alpha| \leqslant m}\left\|X_{\alpha} u\right\|_{L^{p}\left(Q_{T}\right)}+\sum_{r \leqslant k}\left\|X_{t}^{r} u\right\|_{L^{p}\left(Q_{T}\right)} .
$$

The space $W_{p, 0}^{m, k}\left(Q_{T}\right)$ is the closure of $C_{0}^{\infty}\left(\bar{Q}_{T}\right)$ in $W_{p}^{m, k}\left(Q_{T}\right)$, where $C_{0}^{\infty}\left(\bar{Q}_{T}\right)$ is the set of all infinitely differentiable functions in $\bar{Q}_{T}$ vanishing on the parabolic boundary of $Q_{T}$.

Lemma 1 (see [13]). Let $H(\rho)$ be a non-negative increasing function. Suppose that for any $0<\rho<R \leqslant R_{0}=\operatorname{dist}\left(x_{0}, \partial \Omega\right)$,

$$
H(\rho) \leqslant A\left[\left(\frac{\rho}{R}\right)^{a}+\varepsilon\right] H(R)+B R^{b},
$$

where $A, a$ and $b$ are positive constants with $a>b$. Then there exist $\varepsilon_{1}=\varepsilon_{1}(A, a, b)>$ 0 and $c=c(A, a, b)>0$, such that if $\varepsilon<\varepsilon_{1}$, then one has

$$
H(\rho) \leqslant c\left[\left(\frac{\rho}{R}\right)^{b} H(R)+B \rho^{b}\right] .
$$

Lemma 2 (Sobolev-Poincaré inequality, see [9,14]). For any open set $\Omega^{\prime}, \bar{\Omega}^{\prime} \subset \subset$ $\Omega$, there exist positive constants $R_{0}$ and $c$, such that for any $0<R \leqslant R_{0}, B_{R} \subset \Omega$, and $u \in C^{\infty}\left(\overline{B_{R}}\right)$, it holds

$$
\left(\frac{1}{\left|B_{R}\right|} \int_{B_{R}}\left|u-u_{R}\right|^{p^{\prime}} d x\right)^{\frac{1}{p^{\prime}}} \leqslant c R\left(\frac{1}{\left|B_{R}\right|} \int_{B_{R}}|X u|^{p} d x\right)^{\frac{1}{p}},
$$

where $1<p<Q, 1 \leqslant p^{\prime} \leqslant \frac{p Q}{Q-p}, u_{R}=\frac{1}{\left|B_{R}\right|} \int_{B_{R}} u(x) d x, R_{0}$ and $c$ depend on $\Omega$ and $\Omega^{\prime}$.

In particular, if $p=p^{\prime}$, then

$$
\int_{B_{R}}\left|u-u_{R}\right|^{p} d x \leqslant c R^{p} \int_{B_{R}}|X u|^{p} d x .
$$

If $p=2, p^{\prime}=\frac{2 Q}{Q-2}$, then

$$
\left(\frac{1}{\left|B_{R}\right|} \int_{B_{R}}\left|u-u_{R}\right|^{\frac{2 Q}{Q-2}} d x\right)^{\frac{Q-2}{2 Q}} \leqslant c R\left(\frac{1}{\left|B_{R}\right|} \int_{B_{R}}|X u|^{2} d x\right)^{\frac{1}{2}} .
$$

For $u \in C_{0}^{\infty}\left(\overline{B_{R}}\right)$, it follows

$$
\left(\frac{1}{\left|B_{R}\right|} \int_{B_{R}}|u|^{p^{\prime}} d x\right)^{\frac{1}{p^{\prime}}} \leqslant c R\left(\frac{1}{\left|B_{R}\right|} \int_{B_{R}}|X u|^{p} d x\right)^{\frac{1}{p}} .
$$


We need to define a parabolic distance $d_{p}$ corresponding to $d_{X}$. For $(x, t),(y$, $s) \in Q_{T}$, set

$$
d_{p}((x, t),(y, s))=\sqrt{d_{X}(x, y)^{2}+|t-s|} .
$$

Denote a ball under the distance $d_{p}$ :

$$
B_{p}\left(\left(x_{0}, t_{0}\right), R\right)=\left\{(x, t) \in Q_{T}: d_{p}\left(\left(x_{0}, t_{0}\right),(x, t)\right)<R\right\} .
$$

Since $B_{p}\left(\left(x_{0}, t_{0}\right), R\right)$ is a homogeneous space ([1]) and it holds

$$
Q_{R}(z) \subset B_{p}(z, 2 R) \subset Q_{2 R}(z),
$$

we immediately follow the following result, which is the generalization on $Q_{T}$ of results in [10].

Lemma 3 (reverse Hölder inequality). Let $\hat{g}, \hat{f}$ be nonnegative on $Q_{T}$ and satisfy

$$
\hat{g} \in L^{\hat{q}}\left(Q_{T}\right) \text { and } \hat{f} \in L^{r}\left(Q_{T}\right), \quad 1<\hat{q}<r .
$$

Assume that there exist constants $b>0$ and $\theta \in[0,1)$ such that for any $Q_{2 R} \subset Q_{T}$ the following inequality holds

$$
\begin{aligned}
\frac{1}{\left|Q_{R}\right|} \iint_{Q_{R}} \hat{g}^{\hat{q}} d z & \leqslant b\left[\left(\frac{1}{\left|Q_{4 R / 3}\right|} \iint_{Q_{4 R / 3}} \hat{g} d z\right)^{\hat{q}}+\frac{1}{\left|Q_{4 R / 3}\right|} \iint_{Q_{4 R / 3}} \hat{f} \hat{q} d z\right] \\
& +\theta \frac{1}{\left|Q_{4 R / 3}\right|} \iint_{Q_{4 R / 3}} \hat{g}^{\hat{q}} d z,
\end{aligned}
$$

then there exist positive constants $\varepsilon_{0}$ and $\theta_{0}=\theta_{0}\left(\hat{q}, Q_{T}\right)$, such that if $\theta<\theta_{0}$, then $\hat{g} \in L_{l o c}^{\hat{p}}\left(Q_{T}\right)$ for any $\hat{p} \in\left[\hat{q}, \hat{q}+\varepsilon_{0}\right)$, and

$$
\left(\frac{1}{\left|Q_{R}\right|} \iint_{Q_{R}} \hat{g}^{\hat{p}} d z\right)^{\frac{1}{\hat{p}}} \leqslant c\left[\left(\frac{1}{\left|Q_{2 R}\right|} \iint_{Q_{2 R}} \hat{g}^{\hat{q}} d z\right)^{\frac{1}{\hat{q}}}+\left(\frac{1}{\left|Q_{2 R}\right|} \iint_{Q_{2 R}} \hat{f} \hat{p} d z\right)^{\frac{1}{\hat{p}}}\right],
$$

where $c$ and $\varepsilon_{0}$ depend on $b, \hat{q}, \theta$ and $Q$.

Lemma 4 (Jensen inequality). For any bounded open subset $U \subset \mathbb{R}^{n}$, suppose that $f: U \rightarrow \mathbb{R}$ is an integrable function and $\omega: \mathbb{R} \rightarrow \mathbb{R}$ is a concave function. Then

$$
\frac{1}{|U|} \iint_{U} \omega(f) d z \leqslant \omega\left(\frac{1}{|U|} \iint_{U} f d z\right) .
$$




\section{Parabolic CACCioppoli inequality ANd $L^{p}$ EStimates}

In this section, we first establish a parabolic Caccioppoli inequality (Theorem 2) for the weak solution $u$ to (1.1) with the help of the average $\bar{u}(t)$ of $u$ and then get a $L^{p}$ estimates for the gradient of the weak solution (Theorem 3) by using Lemma 3.

Let two cut-off functions $\xi(x)$ and $\eta(t)$ satisfy that for any $0<\rho<R$, $B_{\rho} \subset B_{R} \subset \Omega$,

$$
\begin{gathered}
\xi(x) \in C_{0}^{\infty}\left(B_{R}\right), 0 \leqslant \xi \leqslant 1,|X \xi| \leqslant \frac{c}{R-\rho}, \text { and } \xi=1 \text { in } B_{\rho}, \\
\eta(t)=\left\{\begin{array}{c}
\frac{2 t-2\left(t_{0}-\frac{R^{2}}{2}\right)}{R^{2}-\rho^{2}}, t \in\left(t_{0}-\frac{R^{2}}{2}, t_{0}-\frac{\rho^{2}}{2}\right), \\
1, \quad t \in\left[t_{0}-\frac{\rho^{2}}{2}, t_{0}+\frac{R^{2}}{2}\right] .
\end{array}\right.
\end{gathered}
$$

and set $\frac{1}{\left|B_{R}\right|} \int_{B_{R}} \xi^{2} d x=N_{1}$. Similarly to $[5,12]$, we denote the average on $B_{R}$ of $u(x, t)$ by

$$
\bar{u}(t)=\left(\int_{B_{R}} \xi^{2} d x\right)^{-1} \int_{B_{R}} u \xi^{2} d x=\frac{1}{N_{1}\left|B_{R}\right|} \int_{B_{R}} u \xi^{2} d x
$$

Theorem 2 (parabolic Caccioppoli inequality). Let $u \in W_{2}^{1,1}\left(Q_{T}, \mathbb{R}^{N}\right) \cap L^{\infty}\left(Q_{T}, \mathbb{R}^{N}\right)$ be a weak solution of (1.1). Then for any $0<\rho<R, Q_{R} \subset Q_{T}$, one has

$$
\begin{aligned}
& \sup _{I_{\rho}} \int_{B_{\rho}}|u-\bar{u}(t)|^{2} d x+\iint_{Q_{\rho}}|X u|^{2} d z \\
& \leqslant \frac{c}{(R-\rho)^{2}} \iint_{Q_{R}}|u-\bar{u}(t)|^{2} d z+c \iint_{Q_{R}}\left(\sum_{i}\left|g^{i}\right|^{2}+|f|^{2}\right) d z,
\end{aligned}
$$

where the positive constant $c$ depends on $Q, \delta, L, M$ and $\mu(M)$, $I_{\rho}=\left(t_{0}-\frac{\rho^{2}}{2}, t_{0}+\frac{\rho^{2}}{2}\right]$.

Proof. Noting $B_{\rho} \subset B_{R} \subset \Omega$, multiplying both sides of (1.1) by $(u-\bar{u}(t)) \xi^{2}$ (x) $\eta(t)$, and integrating on $Q_{R}^{\prime}=B_{R}\left(x_{0}\right) \times\left(t_{0}-\frac{R^{2}}{2}, s\right]\left(s \leqslant t_{0}+\frac{R^{2}}{2}\right)$, we get

$$
\begin{aligned}
& \iint_{Q_{R}^{\prime}}\left[u_{t}^{i}+X_{\alpha}^{*}\left(a_{i}^{\alpha \beta} X_{\beta} u^{i}\right)\right]\left(u^{i}-\bar{u}^{i}(t)\right) \xi^{2} \eta d z \\
& =\iint_{Q_{R}^{\prime}}\left[g_{i}+X_{\alpha}^{*} f_{i}^{\alpha}\right]\left(u^{i}-\bar{u}^{i}(t)\right) \xi^{2} \eta d z .
\end{aligned}
$$

By properties of $\xi(x)$ and $\eta(t)$, and the definition of $\bar{u}(t)$, we have

$$
\int_{B_{R}}(u-\bar{u}(t)) \xi^{2} \eta d x=\int_{B_{R}} u \xi^{2} \eta d x-\bar{u}(t) \int_{B_{R}} \xi^{2} \eta d x=0,
$$


GRADIENT ESTIMATES IN MURREY SPACES

859

and

$$
\iint_{Q_{R}} \bar{u}(t)_{t}(u-\bar{u}(t)) \xi^{2} \eta d z=\int_{I_{R}} \bar{u}(t)_{t} d t \int_{B_{R}}(u-\bar{u}(t)) \xi^{2} \eta=0 .
$$

It yields

$$
\begin{aligned}
& \iint_{Q_{R}} u_{t}(u-\bar{u}(t)) \xi^{2} \eta d z=\iint_{Q_{R}}\left(u_{t}-\bar{u}(t)_{t}\right)(u-\bar{u}(t)) \xi^{2} \eta d z \\
& =\iint_{Q_{R}}\left[\left(\frac{1}{2}|u-\bar{u}(t)|^{2} \eta\right)_{t} \xi^{2}-\frac{1}{2}|u-\bar{u}(t)|^{2} \xi^{2} \eta_{t}\right] d z .
\end{aligned}
$$

Since the left and right hand sides of (3.2) have the form

$$
\begin{aligned}
& \iint_{Q_{R}^{\prime}}\left[u_{t}^{i}+X_{\alpha}^{*}\left(a_{i}^{\alpha \beta} X_{\beta} u^{i}\right)\right]\left(u^{i}-\bar{u}^{i}(t)\right) \xi^{2} \eta d z \\
& =\iint_{Q_{R}^{\prime}}\left[\left(\frac{1}{2}\left|u^{i}-\bar{u}^{i}(t)\right|^{2} \eta\right)_{t} \xi^{2}-\frac{1}{2}\left|u^{i}-\bar{u}^{i}(t)\right|^{2} \xi^{2} \eta_{t}+a_{i}^{\alpha \beta} \xi^{2} \eta X_{\alpha} u^{i} X_{\beta} u^{i}\right. \\
& \left.+2 a_{i}^{\alpha \beta}\left(u^{i}-\bar{u}^{i}(t)\right) \xi \eta X_{\alpha} \xi X_{\beta} u^{i}\right] d z
\end{aligned}
$$

and

$$
\begin{aligned}
& \iint_{Q_{R}^{\prime}}\left[g_{i}+X_{\alpha}^{*} f_{i}^{\alpha}\right]\left(u^{i}-\bar{u}^{i}(t)\right) \xi^{2} \eta d z \\
& =\iint_{Q_{R}^{\prime}}\left[g_{i}\left(u^{i}-\bar{u}^{i}(t)\right) \xi^{2} \eta+f_{i}^{\alpha} \xi^{2} \eta X_{\alpha} u^{i}+2 \xi \eta\left(u^{i}-\bar{u}^{i}(t)\right) f_{i}^{\alpha} X_{\alpha} \xi\right] d z,
\end{aligned}
$$

respectively, it follows that (3.2) becomes

$$
\begin{aligned}
& \iint_{Q_{R}^{\prime}}\left[\left(\frac{1}{2}\left|u^{i}-\bar{u}^{i}(t)\right|^{2} \eta\right)_{t} \xi^{2}+a_{i}^{\alpha \beta} \xi^{2} \eta X_{\alpha} u^{i} X_{\beta} u^{i}\right] d z \\
& =\iint_{Q_{R}^{\prime}}\left[\frac{1}{2}\left|u^{i}-\bar{u}^{i}(t)\right|^{2} \xi^{2} \eta_{t}-2 a_{i}^{\alpha \beta}\left(u^{i}-\bar{u}^{i}(t)\right) \xi \eta X_{\alpha} \xi X_{\beta} u^{i}\right] d z \\
& +\iint_{Q_{R}^{\prime}}\left[g_{i}\left(u^{i}-\bar{u}^{i}(t)\right) \xi^{2} \eta+f_{i}^{\alpha} \xi^{2} \eta X_{\alpha} u^{i}\right] d z \\
& +\iint_{Q_{R}^{\prime}}\left[2 \xi \eta\left(u^{i}-\bar{u}^{i}(t)\right) f_{i}^{\alpha} X_{\alpha} \xi\right] d z .
\end{aligned}
$$


By (H1), (H4), $|u| \leqslant M$ and the Young inequality, it implies from (3.3) that

$$
\begin{aligned}
& \iint_{Q_{R}^{\prime}}\left(\frac{1}{2}|u-\bar{u}(t)|^{2} \eta\right)_{t} \xi^{2} d z+\delta \iint_{Q_{R}^{\prime}}|X u|^{2} \xi^{2} \eta d z \\
& \leqslant \iint_{Q_{R}^{\prime}} \frac{1}{2}|u-\bar{u}(t)|^{2} \xi^{2} \eta_{t} d z+\varepsilon \iint_{Q_{R}^{\prime}}|X u|^{2} \xi^{2} \eta d z \\
& +c_{\varepsilon} \iint_{Q_{R}^{\prime}}|u-\bar{u}(t)|^{2}|X \xi|^{2} \eta d z+2 \mu(M) M \iint_{Q_{R}^{\prime}}|X u|^{2} \xi^{2} \eta d z \\
& +\mu(M) c_{\varepsilon} \iint_{Q_{R}^{\prime}} \sum_{i}\left|g^{i}\right|^{2} \xi^{2} \eta d z+\mu(M) \varepsilon \iint_{Q_{R}^{\prime}}|u-\bar{u}(t)|^{2} \xi^{2} \eta d z \\
& +2 c_{\varepsilon} \iint_{Q_{R}^{\prime}}|f|^{2} \xi^{2} \eta d z+\varepsilon \iint_{Q_{R}^{\prime}}|X u|^{2} \xi^{2} \eta d z .
\end{aligned}
$$

Using

$$
\iint_{Q_{R}^{\prime}}\left(\frac{1}{2}|u-\bar{u}(t)|^{2} \eta\right)_{t} \xi^{2} d z=\int_{B_{R}} \frac{1}{2}|u-\bar{u}(s)|^{2} \xi^{2} \eta d x,
$$

it follows by (3.4) that

$$
\begin{aligned}
& \int_{B_{R}} \frac{1}{2}|u-\bar{u}(s)|^{2} \xi^{2} \eta d x+(\delta-2(\varepsilon+\mu(M) M)) \iint_{Q_{R}^{\prime}}|X u|^{2} \xi^{2} \eta d z \\
& \leqslant \iint_{Q_{R}^{\prime}} \frac{1}{2}|u-\bar{u}(t)|^{2} \xi^{2} \eta t d z+c_{\varepsilon} \iint_{Q_{R}^{\prime}}|u-\bar{u}(t)|^{2}|X \xi|^{2} \eta d z \\
& +2 c_{\varepsilon} \iint_{Q_{R}^{\prime}}|f|^{2} \xi^{2} \eta d z+\mu(M) \varepsilon \iint_{Q_{R}^{\prime}}|u-\bar{u}(t)|^{2} \xi^{2} \eta d z \\
& +\mu(M) c_{\varepsilon} \iint_{Q_{R}^{\prime}} \sum_{i}\left|g^{i}\right|^{2} \xi^{2} \eta d z .
\end{aligned}
$$

Because $2 \mu(M) M<\delta$, we can choose a small positive $\varepsilon$ such that $2(\varepsilon+\mu(M)$ $M)<\delta$, and by (3.5) we obtain (3.1). The proof is completed.

Theorem 3. Let $u \in W_{2}^{1,1}\left(Q_{T}, \mathbb{R}^{N}\right) \cap L^{\infty}\left(Q_{T}, \mathbb{R}^{N}\right)$ be a weak solution of (1.1) in $Q_{T}$. Then there exists a positive constants $\varepsilon_{0}$ such that $X u \in L_{l o c}^{p}\left(Q_{T}\right), p \in$ $\left[2,2+\frac{2 Q}{Q+2} \varepsilon_{0}\right)$, and for any $Q_{R} \subset Q_{2 R} \subset Q_{T}$, it holds

$$
\left(\frac{1}{\left|Q_{R}\right|} \iint_{Q_{R}}|X u|^{p} d z\right)^{\frac{1}{p}} \leqslant
$$


$\leqslant c\left[\left(\frac{1}{\left|Q_{2 R}\right|} \iint_{Q_{2 R}}|X u|^{2} d z\right)^{\frac{1}{2}}+\left(\frac{1}{\left|Q_{2 R}\right|} \iint_{Q_{2 R}}\left(\sum_{i}\left|g^{i}\right|^{2}+|f|^{2}\right)^{\frac{p}{2}} d z\right)^{\frac{1}{p}}\right]$

where the positive constant $c$ depends on $Q, \delta, L, M$ and $\mu(M)$.

Proof. Denote $2^{*}=\frac{2 Q}{Q-2}, \tilde{q}=\frac{2 Q}{Q+2}$. Clearly,

$$
\begin{aligned}
& \iint_{Q_{4 R / 5}}|u-\bar{u}(t)|^{2} d z \\
& \leqslant \sup _{I_{4 R / 5}}\left(\int_{B_{4 R / 5}}|u-\bar{u}(t)|^{2} d x\right)^{\frac{1}{2}} \cdot\left(\int_{I_{4 R / 5}}\left(\int_{B_{4 R / 5}}|u-\bar{u}(t)|^{2} d x\right)^{\frac{1}{2}} d t\right) \\
& \equiv A \cdot B .
\end{aligned}
$$

In virtue of (2.2) and (3.1),

$$
\begin{aligned}
A & \leqslant \frac{c}{R}\left(\iint_{Q_{R}}|u-\bar{u}(t)|^{2} d z\right)^{\frac{1}{2}}+c\left(\iint_{Q_{R}}\left(\sum_{i}\left|g^{i}\right|^{2}+|f|^{2}\right) d z\right)^{\frac{1}{2}} \\
& \leqslant c\left(\iint_{Q_{R}}|X u|^{2} d z\right)^{\frac{1}{2}}+c\left(\iint_{Q_{R}}\left(\sum_{i}\left|g^{i}\right|^{2}+|f|^{2}\right) d z\right)^{\frac{1}{2}} .
\end{aligned}
$$

By (2.1), (2.2), (2.3) and the Hölder inequality, we have

$$
\begin{aligned}
B & \leqslant \int_{I_{R}}\left(\int_{B_{R}}|u-\bar{u}(t)|^{\tilde{q}} d x\right)^{\frac{1}{2 \widetilde{q}}}\left(\int_{B_{R}}|u-\bar{u}(t)|^{2^{*}} d x\right)^{\frac{1}{2 \cdot 2^{*}}} d t \\
& \leqslant c R^{\frac{1}{2}} \int_{I_{R}}\left(\int_{B_{R}}|X u|^{\tilde{q}} d x\right)^{\frac{1}{2 \widetilde{q}}}\left(\int_{B_{R}}|X u|^{2} d x\right)^{\frac{1}{4}} d t \\
& \leqslant c R^{\frac{3}{2}-\frac{1}{Q}}\left(\iint_{Q_{R}}|X u|^{\tilde{q}} d z\right)^{\frac{1}{2 \tilde{q}}}\left(\iint_{Q_{R}}|X u|^{2} d z\right)^{\frac{1}{4}} .
\end{aligned}
$$

Inserting (3.8) and (3.9) into (3.7) follows

$$
\begin{aligned}
& \iint_{Q_{4 R / 5}}|u-\bar{u}(t)|^{2} d z \leqslant c R^{\frac{3}{2}-\frac{1}{Q}}\left(\iint_{Q_{R}}|X u|^{2} d z\right)^{\frac{3}{4}} \cdot\left(\iint_{Q_{R}}|X u|^{\tilde{q}} d z\right)^{\frac{1}{2 \tilde{q}}} \\
& +c R^{\frac{3}{2}-\frac{1}{Q}}\left(\iint_{Q_{R}}|X u|^{\tilde{q}} d z\right)^{\frac{1}{2 \tilde{q}}}\left(\iint_{Q_{R}}|X u|^{2} d z\right)^{\frac{1}{4}} \cdot\left(\iint_{Q_{R}}\left(\sum_{i}\left|g^{i}\right|^{2}+|f|^{2}\right) d z\right)^{\frac{1}{2}} \\
& \equiv I+I I .
\end{aligned}
$$


To estimate $I$ and $I I$, it uses the Young inequality to get

$$
\begin{aligned}
I & \leqslant \varepsilon R^{2} \iint_{Q_{R}}|X u|^{2} d z+c_{\varepsilon} R^{-\frac{4}{Q}}\left(\iint_{Q_{R}}|X u|^{\tilde{q}} d z\right)^{\frac{2}{\tilde{q}}}, \\
I I & \leqslant \varepsilon R^{2} \iint_{Q_{R}}|X u|^{2} d z+\varepsilon c_{\varepsilon} R^{-\frac{4}{Q}}\left(\iint_{Q_{R}}|X u|^{\tilde{q}} d z\right)^{\frac{2}{\tilde{q}}} \\
& +c_{\varepsilon} R^{2} \iint_{Q_{R}}\left(\sum_{i}\left|g^{i}\right|^{2}+|f|^{2}\right) d z .
\end{aligned}
$$

Putting (3.11) and (3.12) into (3.10),

$$
\begin{aligned}
\iint_{Q_{4 R / 5}}|u-\bar{u}(t)|^{2} d z & \leqslant \varepsilon R^{2} \iint_{Q_{R}}|X u|^{2} d z+c_{\varepsilon} R^{-\frac{4}{Q}}\left(\iint_{Q_{R}}|X u|^{\tilde{q}} d z\right)^{\frac{2}{\tilde{q}}} \\
& +c_{\varepsilon} R^{2} \iint_{Q_{R}}\left(\sum_{i}\left|g^{i}\right|^{2}+|f|^{2}\right) d z
\end{aligned}
$$

Let us return (3.1) with (3.13), then

$$
\begin{aligned}
\frac{1}{\left|Q_{3 R / 4}\right|} \iint_{Q_{3 R / 4}}|X u|^{2} d z & \leqslant \frac{\varepsilon}{\left|Q_{R}\right|} \iint_{Q_{R}}|X u|^{2} d z+c_{\varepsilon}\left(\frac{1}{\left|Q_{R}\right|} \iint_{Q_{R}}|X u|^{\tilde{q}} d z\right)^{\frac{2}{q}} \\
& +\frac{c_{\varepsilon}}{\left|Q_{R}\right|} \iint_{Q_{R}}\left(\sum_{i}\left|g^{i}\right|^{2}+|f|^{2}\right) d z .
\end{aligned}
$$

Denoting $\hat{g}=|X u|^{\tilde{q}}\left(\hat{q}=\frac{2}{\tilde{q}}=\frac{Q+2}{Q}>1\right), \hat{f}=\left(\sum_{i}\left|g^{i}\right|^{2}+|f|^{2}\right)^{\frac{Q}{Q+2}}, \theta=\varepsilon$, the above inequality is

$$
\begin{aligned}
\frac{1}{\left|Q_{3 R / 4}\right|} \iint_{Q_{3 R / 4}} \hat{g}^{\hat{q}} d z & \leqslant c\left[\left(\frac{1}{\left|Q_{R}\right|} \iint_{Q_{R}} \hat{g} d z\right)^{\hat{q}}+\frac{1}{\left|Q_{R}\right|} \iint_{Q_{R}} \hat{f^{\hat{q}}} d z\right] \\
& +\frac{\theta}{\left|Q_{R}\right|} \iint_{Q_{R}} \hat{g}^{\hat{q}} d z .
\end{aligned}
$$

By Lemma 3, we know that there exist positive constant $c$ and $\varepsilon_{0}$, such that for $\hat{p} \in\left[\hat{q}, \hat{q}+\varepsilon_{0}\right)$,

$$
\left(\frac{1}{\left|Q_{R}\right|} \iint_{Q_{R}}|X u|^{\hat{p} \tilde{q}} d z\right)^{\frac{1}{\hat{p}}} \leqslant
$$




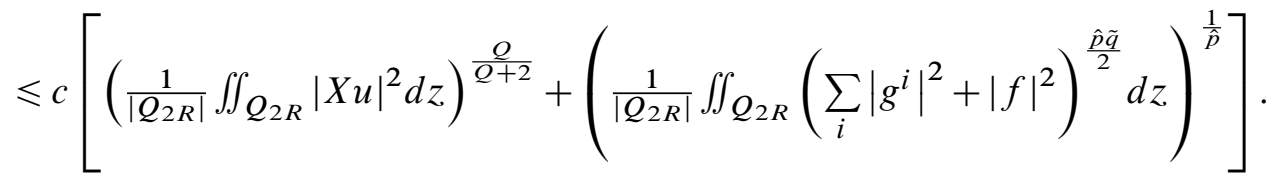

Setting $p=\hat{p} \tilde{q} \in\left[2,2+\frac{2 Q}{Q+2} \varepsilon_{0}\right)$, the proof is finished.

\section{HomogeneOUS PARABOLIC SYSTEM}

In this section, we study the following homogeneous parabolic system

$$
u_{t}^{i}+X_{\alpha}^{*}\left(a_{i}^{\alpha \beta}(z, u) X_{\beta} u^{i}\right)=0 .
$$

For convenience, we divide (4.1) into two parts. Concretely, let $v \in W_{2}^{1,1} \cap L^{\infty}$ be a weak solution of the following constant coefficients system:

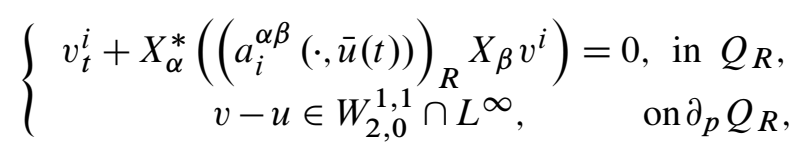

then $w=u-v$ satisfies:

$$
\left\{\begin{array}{cc}
w_{t}^{i}+X_{\alpha}^{*}\left(\left(a_{i}^{\alpha \beta}(\cdot, \bar{u}(t))\right)_{R} X_{\beta} w^{i}\right) & \\
=X_{\alpha}^{*}\left(\left(\left(a_{i}^{\alpha \beta}(\cdot, \bar{u}(t))\right)_{R}-a_{i}^{\alpha \beta}(z, u)\right) X_{\beta} u^{i}\right), & \text { in } Q_{R}, \\
w \in W_{2,0}^{1,1} \cap L^{\infty}, & \text { on } \partial_{p} Q_{R},
\end{array}\right.
$$

where $\left(a_{i}^{\alpha \beta}(\cdot, \bar{u}(t))\right)_{R}=\frac{1}{\left|Q_{R}\right|} \iint_{Q_{R}} a_{i}^{\alpha \beta}(z, \bar{u}(t)) d z$.

Lemma 5 ([7]). Let $v \in W_{2}^{1,1}\left(Q_{T}, \mathbb{R}^{N}\right) \cap L^{\infty}\left(Q_{T}, \mathbb{R}^{N}\right)$ be a weak solution of (4.2). Then for any $0<\rho<R, Q_{R} \subset \subset Q_{T}$, one has

$$
\iint_{Q_{\rho}}|X v|^{2} d z \leqslant c\left(\frac{\rho}{R}\right)^{Q+2} \iint_{Q_{R}}|X v|^{2} d z
$$

Theorem 4. Let $u \in W_{2}^{1,1}\left(Q_{T}, \mathbb{R}^{N}\right) \cap L^{\infty}\left(Q_{T}, \mathbb{R}^{N}\right)$ be a weak solution of (4.1). Then for any $0<\rho<R, Q_{R} \subset \subset Q_{T}$, there exists $\varepsilon_{0}>0$, such that for any $p \in$ $\left[2,2+\frac{2 Q}{Q+2} \varepsilon_{0}\right), \frac{p-2}{p}(Q+2)<\mu<Q+2$, we have

$$
\iint_{Q_{\rho}}|X u|^{p} d z \leqslant c\left(\frac{\rho}{R}\right)^{\frac{2(Q+2)-p(Q+2-\mu)}{2}} \iint_{Q_{R}}|X u|^{p} d z .
$$

Proof. When $\frac{1}{2} R \leqslant \rho<R$, the result is clearly true. Now it is enough to treat the case $\rho<\frac{1}{2} R$.

Multiplying both sides of (4.3) by $w^{i}$ and integrating on $Q_{R}$, we obtain

$$
\iint_{Q_{R}}\left(w_{t}^{i} w^{i}+\left(a_{i}^{\alpha \beta}(\cdot, \bar{u}(t))\right)_{R} X_{\beta} w^{i} X_{\alpha} w^{i}\right) d z
$$




$$
=\iint_{Q_{R}}\left(\left(a_{i}^{\alpha \beta}(\cdot, \bar{u}(t))\right)_{R}-a_{i}^{\alpha \beta}(z, u)\right) X_{\beta} u^{i} X_{\alpha} w^{i} d z .
$$

Noting $\iint_{Q_{R}} w_{t}^{i} w^{i} d z=\int_{B_{R}} d x \int_{t_{0}-R^{2} / 2}^{t_{0}+R^{2} / 2} w^{i} d w^{i} \geqslant 0$ and (H1), we get

$$
\begin{aligned}
\delta \iint_{Q_{R}}|X w|^{2} d z & \leqslant \iint_{Q_{R}}\left|\left(a_{i}^{\alpha \beta}(\cdot, \bar{u}(t))\right)_{R}-a_{i}^{\alpha \beta}(z, \bar{u}(t))\right||X u||X w| d z \\
& +\iint_{Q_{R}}\left|a_{i}^{\alpha \beta}(z, \bar{u}(t))-a_{i}^{\alpha \beta}(z, u)\right||X u||X w| d z \\
& =I+I I .
\end{aligned}
$$

Because $a_{i}^{\alpha \beta}(z, u)$ satisfies (H2), it follows

$$
\begin{aligned}
& \iint_{Q_{R}}\left|a_{i}^{\alpha \beta}(z, \bar{u}(t))-\left(a_{i}^{\alpha \beta}(\cdot, \bar{u}(t))\right)_{R}\right|^{\frac{2 p}{p-2}} d z \\
& \leqslant c \iint_{Q_{R}}\left|a_{i}^{\alpha \beta}(z, \bar{u}(t))-\left(a_{i}^{\alpha \beta}(\cdot, \bar{u}(t))\right)_{R}\right| d z \leqslant c\left|Q_{R}\right| \eta_{R}\left(a_{i}^{\alpha \beta}\right) .
\end{aligned}
$$

By the Young inequality and the Hölder inequality, we have

$$
\begin{aligned}
I & \leqslant c_{\varepsilon}\left(\iint_{Q_{R}}\left|a_{i}^{\alpha \beta}(z, \bar{u}(t))-\left(a_{i}^{\alpha \beta}(\cdot, \bar{u}(t))\right)_{R}\right|^{\frac{2 p}{p-2}} d z\right)^{\frac{p-2}{p}}\left(\iint_{Q_{R}}|X u|^{p} d z\right)^{\frac{2}{p}} \\
& +\varepsilon \iint_{Q_{R}}|X w|^{2} d z \\
& \leqslant c_{\varepsilon}\left(\left|Q_{R}\right| \eta_{R}\left(a_{i}^{\alpha \beta}\right)\right)^{\frac{p-2}{p}}\left(\iint_{Q_{R}}|X u|^{p} d z\right)^{\frac{2}{p}}+\varepsilon \iint_{Q_{R}}|X w|^{2} d z
\end{aligned}
$$

By the Young inequality, (H3), Lemma 4 and the Hölder inequality, it shows

$$
\begin{aligned}
I I & \leqslant c_{\varepsilon} \iint_{Q_{R}}\left|a_{i}^{\alpha \beta}(z, \bar{u}(t))-a_{i}^{\alpha \beta}(z, u)\right|^{2}|X u|^{2} d z+\varepsilon \iint_{Q_{R}}|X w|^{2} d z \\
& \leqslant c_{\varepsilon} \iint_{Q_{R}} \omega^{2}\left(|u-\bar{u}(t)|^{2}\right)|X u|^{2} d z+\varepsilon \iint_{Q_{R}}|X w|^{2} d z \\
& \leqslant c_{\varepsilon}\left(\iint_{Q_{R}}\left(\omega\left(|u-\bar{u}(t)|^{2}\right)\right)^{\frac{2 p}{p-2}} d z\right)^{\frac{p-2}{p}}\left(\iint_{Q_{R}}|X u|^{p} d z\right)^{\frac{2}{p}} \\
& +\varepsilon \iint_{Q_{R}}|X w|^{2} d z
\end{aligned}
$$




$$
\begin{aligned}
& \leqslant c_{\varepsilon}\left(\left|Q_{R}\right| \omega\left(\frac{1}{\left|Q_{R}\right|} \iint_{Q_{R}}|u-\bar{u}(t)|^{2} d z\right)\right)^{\frac{p-2}{p}}\left(\iint_{Q_{R}}|X u|^{p} d z\right)^{\frac{2}{p}} \\
& +\varepsilon \iint_{Q_{R}}|X w|^{2} d z .
\end{aligned}
$$

Inserting (4.6) and (4.7) into (4.5), we have

$$
\begin{aligned}
& \delta \iint_{Q_{R}}|X w|^{2} d z \\
& \leqslant c_{\varepsilon}\left|Q_{R}\right|\left[\left(\eta_{R}\left(a_{i}^{\alpha \beta}\right)\right)^{\frac{p-2}{p}}+\left(\omega\left(\frac{1}{\left|Q_{R}\right|} \iint_{Q_{R}}|u-\bar{u}(t)|^{2} d z\right)\right)^{\frac{p-2}{p}}\right] \\
& \cdot\left(\frac{1}{\left|Q_{R}\right|} \iint_{Q_{R}}|X u|^{p} d z\right)^{\frac{2}{p}}+2 \varepsilon \iint_{Q_{R}}|X w|^{2} d z .
\end{aligned}
$$

Let $G\left(z_{0}, R\right)=\left(\eta_{R}\left(a_{i}^{\alpha \beta}\right)\right)^{\frac{p-2}{p}}+\left(\omega\left(\frac{1}{\left|Q_{R}\right|} \iint_{Q_{R}}|u-\bar{u}(t)|^{2} d z\right)\right)^{\frac{p-2}{p}}$ and choose $\varepsilon$ small enough such that $\delta-2 \varepsilon>0$, then the above can be rewritten as

$$
\iint_{Q_{R}}|X w|^{2} d z \leqslant c G\left(z_{0}, R\right)\left|Q_{R}\right|\left(\frac{1}{\left|Q_{R}\right|} \iint_{Q_{R}}|X u|^{p} d z\right)^{\frac{2}{p}} .
$$

By the above, Lemma 5 and the Hölder inequality, we know that for any $\rho<\frac{1}{2} R$,

$$
\begin{aligned}
& \iint_{Q_{2 \rho}}|X u|^{2} d z \leqslant c\left(\frac{\rho}{R}\right)^{Q+2} \iint_{Q_{R}}|X v|^{2} d z+c \iint_{Q_{R}}|X w|^{2} d z \\
& \leqslant c\left(\frac{\rho}{R}\right)^{Q+2} \iint_{Q_{R}}|X u|^{2} d z+c \iint_{Q_{R}}|X w|^{2} d z \\
& \leqslant c\left|Q_{R}\right|\left[\left(\frac{\rho}{R}\right)^{Q+2}+G\left(z_{0}, R\right)\right]\left(\frac{1}{\left|Q_{R}\right|} \iint_{Q_{R}}|X u|^{p} d z\right)^{\frac{2}{p}} .
\end{aligned}
$$

Due to Theorem 3 with $g_{i}=f=0$ and (4.8), it holds

$$
\begin{aligned}
& \iint_{Q_{\rho}}|X u|^{p} d z \leqslant c\left|Q_{\rho}\right|\left(\frac{1}{\left|Q_{2 \rho}\right|} \iint_{Q_{2 \rho}}|X u|^{2} d z\right)^{\frac{p}{2}} \\
& \leqslant c\left(\left(\frac{\rho}{R}\right)^{Q+2}+G\left(z_{0}, R\right)\right)^{\frac{p}{2}}\left(\frac{\left|Q_{R}\right|}{\left|Q_{\rho}\right|}\right)^{\frac{p-2}{2}} \iint_{Q_{R}}|X u|^{p} d z .
\end{aligned}
$$


Taking $H(\rho)=\left(\left|Q_{\rho}\right|^{\frac{p-2}{2}} \iint_{Q_{\rho}}|X u|^{p} d z\right)^{\frac{2}{p}}, H(R)=\left(\left|Q_{R}\right|^{\frac{p-2}{2}} \iint_{Q_{R}}|X u|^{p} d z\right)^{\frac{2}{p}}$, $a=Q+2, B=0$. We have by Lemma 1 with $b=\mu\left(\frac{p-2}{p}(Q+2)<\mu<Q+2\right)$ that

$$
\left(\left|Q_{\rho}\right|^{\frac{p-2}{2}} \iint_{Q_{\rho}}|X u|^{p} d z\right)^{\frac{2}{p}} \leqslant c\left(\frac{\rho}{R}\right)^{\mu}\left(\left|Q_{R}\right|^{\frac{p-2}{2}} \iint_{Q_{R}}|X u|^{p} d z\right)^{\frac{2}{p}}
$$

Inserting $\frac{\left|Q_{R}\right|}{\left|Q_{\rho}\right|} \leqslant c\left(\frac{\rho}{R}\right)^{-Q-2}$ into the above, we get the result.

\section{PROOFS OF THE MAIN RESUlts}

In this section, we will prove Theorem 1. Let $v \in W_{2}^{1,1} \cap L^{\infty}$ be a weak solution of the following system:

$$
\left\{\begin{array}{c}
v_{t}^{i}+X_{\alpha}^{*}\left(a_{i}^{\alpha \beta}(\cdot, \bar{u}(t)) X_{\beta} v^{i}\right)=0, \text { in } Q_{R}, \\
v-u \in W_{2,0}^{1,1} \cap L^{\infty}, \quad \text { on } \partial_{p} Q_{R},
\end{array}\right.
$$

then $w=u-v$ satisfies:

$$
\left\{\begin{array}{cr}
w_{t}^{i}+X_{\alpha}^{*}\left(a_{i}^{\alpha \beta}(\cdot, \bar{u}(t)) X_{\beta} w^{i}\right)=g_{i}+X_{\alpha}^{*} f_{i}^{\alpha}, & \text { in } Q_{R}, \\
w \in W_{2,0}^{1,1} \cap L^{\infty}, & \text { on } \partial_{p} Q_{R} .
\end{array}\right.
$$

Lemma 6. Let $w \in W_{2,0}^{1,1}\left(Q_{T}, \mathbb{R}^{N}\right) \cap L^{\infty}\left(Q_{T}, \mathbb{R}^{N}\right)$ be a weak solution of (5.2) in $Q_{T}$. Then there exists a positive constant $\varepsilon_{0}$, such that $X w \in L_{l o c}^{p}\left(Q_{T}\right), p \in$ $\left[2,2+\frac{2 Q}{Q+2} \varepsilon_{0}\right)$, and it holds

$$
\iint_{Q_{R}}|X w|^{p} d z \leqslant c \iint_{Q_{2 R}}\left(\sum_{i}\left|g^{i}\right|^{p}+|f|^{p}\right) d z+c \iint_{Q_{2 R}}|X u|^{p} d z,
$$

for any $Q_{2 R} \subset \subset Q_{T}$.

Proof. Since $w \in W_{2,0}^{1,1}\left(Q_{T}, \mathbb{R}^{N}\right) \cap L^{\infty}\left(Q_{T}, \mathbb{R}^{N}\right)$, there exists a constant $M_{1}>0$, such that $|w| \leqslant M_{1}$.

Multiplying both sides of (1.1) by $w^{i}$ and integrating on $Q_{2 R}$ leads to

$$
\iint_{Q_{2 R}}\left[w_{t}^{i} w^{i}+a_{i}^{\alpha \beta} X_{\beta} w^{i} X_{\alpha} w^{i}\right] d z=\iint_{Q_{2 R}}\left[g_{i} w^{i}+f_{i}^{\alpha} X_{\alpha} w^{i}\right] d z .
$$

Since

$$
\iint_{Q_{2 R}} w_{t} w d z=\int_{B_{2 R}} d x \int_{I_{2 R}} w \frac{\partial w}{\partial t} d t=\int_{B_{2 R}} \frac{1}{2} w^{2} d x \geqslant 0,
$$


we have by (H1), (H4), the Young inequality and (2.4) that

$$
\begin{aligned}
& \delta \iint_{Q_{2 R}}|X w|^{2} d z \leqslant \iint_{Q_{2 R}} w_{t} w d z+\delta \iint_{Q_{2 R}}|X w|^{2} d z \\
& \leqslant \iint_{Q_{2 R}} \mu(M)\left(|X u|^{2}+\sum_{i}\left|g^{i}\right|\right)|w| d z+c_{\varepsilon} \iint_{Q_{2 R}}|f|^{2} d z+\varepsilon \iint_{Q_{2 R}}|X w|^{2} d z \\
& \leqslant \mu(M) M_{1} \iint_{Q_{2 R}}|X u|^{2} d z+c \iint_{Q_{2 R}}\left(\sum_{i}\left|g^{i}\right|^{2}+|f|^{2}\right) d z+2 \varepsilon \iint_{Q_{2 R}}|X w|^{2} d z,
\end{aligned}
$$

where $\varepsilon$ is small enough such that $\delta-2 \varepsilon>0$. It gets

$$
\iint_{Q_{2 R}}|X w|^{2} d z \leqslant c \iint_{Q_{2 R}}|X u|^{2} d z+c \iint_{Q_{2 R}}\left(\sum_{i}\left|g^{i}\right|^{2}+|f|^{2}\right) d z .
$$

Using the above estimate into (3.6) and the Hölder inequality, it follows

$$
\begin{aligned}
& \frac{1}{\left|Q_{R}\right|} \iint_{Q_{R}}|X w|^{p} d z \\
& \leqslant c\left[\frac{1}{\left|Q_{2 R}\right|} \iint_{Q_{2 R}}|X u|^{2} d z+\frac{1}{\left|Q_{2 R}\right|} \iint_{Q_{2 R}}\left(\sum_{i}\left|g^{i}\right|^{2}+|f|^{2}\right) d z\right]^{\frac{p}{2}} \\
& +\frac{c}{\left|Q_{2 R}\right|} \iint_{Q_{2 R}}\left(\sum_{i}\left|g^{i}\right|^{p}+|f|^{p}\right) d z \\
& \leqslant \frac{c}{\left|Q_{2 R}\right|} \iint_{Q_{2 R}}|X u|^{p} d z+\frac{c}{\left|Q_{2 R}\right|} \iint_{Q_{2 R}}\left(\sum_{i}\left|g^{i}\right|^{p}+|f|^{p}\right) d z .
\end{aligned}
$$

Hence the conclusion is proved.

Theorem 5. Suppose that assumptions (H1)-(H4) hold. Let $u \in W_{2}^{1,1}\left(Q_{T}, \mathbb{R}^{N}\right) \cap$ $L^{\infty}\left(Q_{T}, \mathbb{R}^{N}\right)$ be a weak solution of (1.1). Then there exists a positive constant $\varepsilon_{0}$, such that for any $p \in\left[2,2+\frac{2 Q}{Q+2} \varepsilon_{0}\right)$, we have

$$
X u \in L_{X, l o c}^{p, \lambda}\left(Q_{T}, \mathbb{R}^{q n}\right) .
$$

Moreover, there exists a constant $R_{0} \leqslant d_{0}$ such that for any $\rho$ and $R, 0<\rho<R \leqslant R_{0}$, $Q_{2 R} \subset \subset Q_{T}$, one has

$$
\|X u\|_{L_{X}^{p, \lambda}\left(Q_{\rho}\right)}^{p} \leqslant c\left[\|X u\|_{L^{p}\left(Q_{R}\right)}^{p}+\sum_{i}\left\|g^{i}\right\|_{L_{X}^{p, \lambda}}^{p}+\|f\|_{L_{X}^{p, \lambda}}^{p}\right] .
$$


Proof. By Lemma 6 and Theorem 4, it shows

$$
\begin{aligned}
& \iint_{Q_{\rho}}|X u|^{p} d z \leqslant c \iint_{Q_{\rho}}|X v|^{p} d z+c \iint_{Q_{\rho}}|X w|^{p} d z \\
& \leqslant c\left(\frac{\rho}{R}\right)^{\frac{2(Q+2)-p(Q+2-\mu)}{2}} \iint_{Q_{R}}|X u|^{p} d z+c \iint_{Q_{2 R}}\left(\sum_{i}\left|g^{i}\right|^{p}+|f|^{p}\right) d z \\
& +c \iint_{Q_{2 R}}|X u|^{p} d z \\
& \leqslant\left[c\left(\frac{\rho}{R}\right)^{\frac{2(Q+2)-p(Q+2-\mu)}{2}}+c\right] \iint_{Q_{2 R}}|X u|^{p} d z \\
& +c R^{Q+2-\lambda}\left(\sum_{i}\left\|g^{i}\right\|_{L_{X}^{p, \lambda}}^{p}+\|f\|_{L_{X}^{p, \lambda}}^{p}\right) .
\end{aligned}
$$

Denote $H(\rho)=\iint_{Q_{\rho}}|X u|^{p} d z, H(R)=\iint_{Q_{R}}|X u|^{p} d z, a=\frac{2(Q+2)-p(Q+2-\mu)}{2}$, $b=Q+2-\lambda, B=c\left(\sum_{i}\left\|g^{i}\right\|_{L_{X}^{p, \lambda}}^{p}+\|f\|_{L_{X}^{p, \lambda}}^{p}\right), 0<\lambda<Q+2$. Then there exists $\mu, Q+2-\frac{2 \lambda}{p}<\mu<Q+2$, such that $a>b$. By Lemma 1, we have

$$
\begin{aligned}
& \iint_{Q_{\rho}}|X u|^{p} d z \\
& \leqslant c\left[\left(\frac{\rho}{R}\right)^{Q+2-\lambda} \iint_{Q_{2 R}}|X u|^{p} d z+\rho^{Q+2-\lambda}\left(\sum_{i}\left\|g^{i}\right\|_{L_{X}^{p, \lambda}}^{p}+\|f\|_{L_{X}^{p, \lambda}}^{p}\right)\right] .
\end{aligned}
$$

This proof is completed.

Proof. (of Theorem 1) By Theorem 5 and the cutoff function technique, it is easy to see that Theorem 1 is true.

\section{ACKNOWLEDGEMENT}

The author is sincerely grateful to PengCheng Niu for giving some useful discussions.

\section{REFERENCES}

[1] M. Bramanti and L. Brandolini, "Schauder estimates for parabolic nondivergence operators of Hörmander type,” J. Differ. Equations, vol. 234, no. 1, pp. 177-245, 2007.

[2] S. Campanato, "Equazioni paraboliche del secondo ordine e spazi $\mathfrak{L}^{2, \theta}(\Omega, \delta)$," Ann. Mat. Pura Appl. (4), vol. 73, pp. 55-102, 1966.

[3] S. Campanato, " $L^{p}$ regularity for weak solutions of parabolic systems," Ann. Sc. Norm. Super. Pisa, Cl. Sci., IV. Ser., vol. 7, pp. 65-85, 1980. 
[4] G. Di Fazio and M. S. Fanciullo, "Gradient estimates for elliptic systems in Carnot-Carathéodory spaces," Commentat. Math. Univ. Carol., vol. 43, no. 4, pp. 605-618, 2002.

[5] H. Dong and D. Kim, "Global regularity of weak solutions to quasilinear elliptic and parabolic equations with controlled growth," Commun. Partial Differ. Equations, vol. 36, no. 10-12, pp. 1750-1777, 2011.

[6] Y. Dong and P. Niu, "Estimates in Morrey spaces and Hölder continuity for weak solutions to degenerate elliptic systems," Manuscr. Math., vol. 138, no. 3-4, pp. 419-437, 2012.

[7] Y. Dong, M. C. Zhu, and P. C. Niu, "Estimates in generalized Morrey spaces for weak solutions to divergence degenerate parabolic systems," arXiv: 1109. 1929vl Math. AP, 2011.

[8] D. Gao, P. Niu, and J. Wang, "Partial regularity for degenerate subelliptic systems associated with Hörmander's vector fields," Nonlinear Anal., Theory Methods Appl., Ser. A, Theory Methods, vol. 73, no. 10, pp. 3209-3223, 2010.

[9] N. Garofalo and D.-M. Nhieu, "Isoperimetric and Sobolev inequalities for Carnot-Carathéodory spaces and the existence of minimal surfaces," Commun. Pure Appl. Math., vol. 49, no. 10, pp. 1081-1144, 1996.

[10] U. Gianazza, "Regularity for nonlinear equations involving square Hörmander operators," Nonlinear Anal., Theory Methods Appl., Ser. A, Theory Methods, vol. 23, no. 1, pp. 49-73, 1994.

[11] M. Giaquinta and E. Giusti, "Partial regularity for the solutions to nonlinear parabolic systems," Ann. Mat. Pura Appl. (4), vol. 97, pp. 253-266, 1973.

[12] M. Giaquinta and M. Struwe, "On the partial regularity of weak solutions on nonlinear parabolic systems," Math. Z., vol. 179, pp. 437-451, 1982.

[13] Q. Han and F. Lin, Elliptic partial differential equations, ser. Courant Lecture Notes in Mathematics. New York; Providence: NY: Courant Institute of Mathematical Sciences; RI: American Mathemtical Society (AMS), 1997, vol. 1.

[14] G. Lu, "The sharp Poincaré inequality for free vector fields: An endpoint result," Rev. Mat. Iberoam., vol. 10, no. 2, pp. 453-466, 1994.

[15] M. Mcbride, "Estimates in the generalized Morrey spaces for linear parabolic systems," arXiv: 0811. 3360 Math. AP, 2010

[16] A. Nagel, E. M. Stein, and S. Wainger, "Balls and metrics defined by vector fields. I: Basic properties," Acta Math., vol. 155, pp. 103-147, 1985.

[17] M. Struwe, "On the Hölder continuity of bounded weak solutions of quasilinear parabolic systems," Manuscr. Math., vol. 35, pp. 125-145, 1981.

[18] S. Tang and P. Niu, "Morrey estimates for parabolic nondivergence operators of Hörmander type," Rend. Semin. Mat. Univ. Padova, vol. 123, pp. 91-129, 2010.

[19] J. Wang, P. Niu, and X. Cui, " $l p$ estimates for weak solutions to nonlinear sub-elliptic systems related to Hörmander's vector fields," Kyushu J. Math., vol. 63, pp. 301-314, 2009.

[20] C.-J. Xu and C. Zuily, "Higher interior regularity for quasilinear subelliptic systems," Calc. Var. Partial Differ. Equ., vol. 5, no. 4, pp. 323-343, 1997.

\section{Author's address}

\section{Yan Dong}

Northwestern Polytechnical University, Department of Applied Mathematics; Key Laboratory of Space Applied Physics and Chemistry, Ministry of Education, Xi'an, Shaanxi, 710129, China

E-mail address: dongyan@mail.nwpu.edu.cn 